\title{
Selenoprotein expression is regulated at multiple levels in prostate cells
}

\author{
Cheryl M Rebsch ${ }^{1}$, Frank J Penna III ${ }^{1}$, Paul R Copeland $d^{1,2}$ \\ ${ }^{I}$ Department of Molecular Genetics, Microbiology and Immunology; ${ }^{2}$ The Cancer Institute of New Jersey, UMDNJ - Robert Wood \\ Johnson Medical School, 675 Hoes Lane, Piscataway, NJ 08854, USA
}

Selenium supplementation in a population with low basal blood selenium levels has been reported to decrease the incidence of several cancers including prostate cancer. Based on the clinical findings, it is likely that the antioxidant function of one or more selenoproteins is responsible for the chemopreventive effect, although low molecular weight seleno-compounds have also been posited to selectively induce apoptosis in transformed cells. To address the effects of selenium supplementation on selenoprotein expression in prostate cells, we have undertaken an analysis of antioxidant selenoprotein expression as well as selenium toxicity in non-tumorigenic prostate epithelial cells (RWPE-1) and prostate cancer cells (LNCaP and PC-3). Our results show that two of the glutathione peroxidase family members (GPX1 and GPX4) are highly induced by supplemental selenium in prostate cancer cells but only slightly induced in RWPE-1 cells. In addition, GPX1 levels are dramatically lower in PC-3 cells as compared to RWPE-1 or LNCaP cells. GPX2 protein and mRNA, however, are only detectable in RWPE-1 cells. Of the three selenium compounds tested (sodium selenite, sodium selenate and selenomethionine), only sodium selenite shows toxicity in a physiological range of selenium concentrations. Notably and in contrast to previous studies, RWPE-1 cells were significantly more sensitive to selenite than either of the prostate cancer cell lines. These results demonstrate that selenoproteins and selenium metabolism are regulated at multiple levels in prostate cells.

Cell Research (2006) 16:940-948. doi: 10.1038/sj.cr.7310117; published online 12 December 2006

Keywords: prostate, selenium, selenoproteins, glutathione peroxidase, selenocysteine

\section{Introduction}

In 1996, Clark et al. [1] reported a dramatic decrease in the incidence of prostate, lung and colorectal cancer in skin cancer patients who supplemented their diets with $200 \mu \mathrm{g}$ of selenium in the form of selenized yeast. Further analysis showed that only the patients with low baseline selenium benefited from the regimen [2]. Selenium supplementation did not prevent the recurrence of skin cancer; in fact, recent follow-up has shown that squamous cell carcinoma and total non-melanoma skin cancer incidence increased [3], highlighting the potential danger of indiscriminant

Correspondence: Paul R Copeland

Tel: +1-732-235-4670; Fax +1-732-235-5223;

E-mail: paul.copeland@umdnj.edu

Received 22 September 2006; revised 1 November 2006; accepted 3 November 2006; published online 12 December 2006 selenium supplementation. The mechanism of selenium chemoprevention remains unknown, but two theories have developed. First, small molecule selenium metabolites have been postulated to selectively promote apoptosis in transformed prostate epithelium [4-6]. Alternatively, or additionally, it has been proposed that selenium supplementation increases the levels of anti-oxidant selenoproteins and thereby prevents the DNA damage that could lead to cell transformation $[7,8]$. The majority of work to date has investigated the role of selenium in apoptosis, but several studies have also implicated selenoproteins as key players in chemoprevention. Some studies have established the correlation between the incidence of prostate cancer and the inheritance of an attenuating polymorphism in the gene encoding Sep15, a selenoprotein proposed to be involved in protein folding $[9,10]$. Both Sep 15 and glutahione peroxidase 1 (GPX1) levels have been reported to be significantly reduced in prostate cancer $[11,12]$. In addition, 
the recent use of a mouse model has suggested that loss of a select group of selenoproteins in animals expressing mutant selenocysteine $(\mathrm{Sec})-\mathrm{RRNA}^{[\mathrm{Ser}] \mathrm{Sec}}$ leads to increased incidence of colorectal cancer [13]. While these studies cannot distinguish between selenoprotein regulation before or after transformation, these data in combination with the clinical observation that selenium supplementation has only been shown to be effective in humans when baseline selenium is at the level where selenoprotein production is regulated [2], suggest that the induction of selenoprotein activity seems to be a likely mechanism for selenium chemoprevention.

Selenoprotein synthesis is a complex and highly regulated process. Selenium is incorporated as Sec at specific UGA codons in 25 human proteins (reviewed in [14-16]). Selenoprotein mRNAs all contain a cis-element within the 3' untranslated region (termed the Sec insertion sequence (SECIS) element) that is required for Sec incorporation. In addition, at least two protein factors are required for the insertion of Sec-tRNA ${ }^{[\mathrm{Ser}] \mathrm{Sec}}$ into the ribosomal A-site: a SECIS element binding protein (SBP2) and the Sec-specific elongation factor. Surprisingly, a thorough examination of the effects of selenium supplementation on selenoprotein expression in prostate cells is lacking. In this study, we used the three common dietary supplements, sodium selenite, sodium selenate and selenomethionine (SeMet), to supplement the growth medium for "normal" prostate epithelial cells (RWPE-1; cells derived from normal prostate epithelia that were immortalized with human papolloma virus [17]) as well as androgen sensitive (LNCaP) and androgen insensitive (PC-3) prostate cancer cell lines. Because of their potential role in preventing transformation caused by DNA damage, we have chosen to focus on the three major intracellular antioxidant selenoproteins found in epithelial cells, GPX1, GPX2 and GPX4. GPX1 levels are dramatically upregulated by selenium supplementation in prostate cancer but not RWPE-1 cells. In addition, GPX1 is elevated in LNCaP and reduced in PC-3 relative to RWPE-1 cells and importantly, PC-3 cells are limited in their ability to utilize organic selenium for selenoprotein expression. We also observed dramatic differences in toxicity and selenoprotein expression as a function of both selenium compound and cell type. Surprisingly, we did not observe selective toxicity in cancer cells as predicted by the apoptosis model of chemoprevention. In fact, RWPE-1 cells are notably more sensitive to toxic levels of selenium. Overall, these studies point to dramatic differences in selenium metabolism and selenoprotein production in prostate cell lines.

\section{Materials and Methods}

\section{Chemicals and reagents}

Sodium selenite, sodium selenate and seleno-L-methionine
(Sigma), RPMI 1640, fetal bovine serum, and L-glutamine (Cellgro Mediatech Inc.), MCDB-151 (Biosource), epidermal growth factor (EGF) and bovine pituitary extract (BPE) (Invitrogen).

\section{Cells and cell culture}

LNCaP and RWPE- 1 cells were obtained from the American Type Culture Collection and PC-3 cells were a gift from Arnold B Rabson (UMDNJ-RWJMS, Cancer Institute of New Jersey). RWPE-1 cells were grown in MCDB-151 medium containing $8 \mathrm{mM} \mathrm{L}$-glutamine, $12.94 \mathrm{mg} / \mathrm{ml} \mathrm{BPE}$ and $1 \mathrm{mg} / \mathrm{ml}$ EGF. LNCaP and PC-3 cells were grown in RPMI 1640 medium containing $10 \%$ fetal bovine serum. RWPE-1, PC-3 and LNCaP cells were seeded at 5000 cells $/ \mathrm{cm}^{2}$ into six-well cell culture cluster plates containing medium with either sodium selenite, SeMet or sodium selenate at concentrations indicated in the figure legends. For the testing of BPE-induced expression of GPX1, PC-3 or LNCaP, cells were grown in the presence or absence of $12.94 \mathrm{mg} / \mathrm{ml} \mathrm{BPE}$ or in the presence of $50 \mathrm{nM}$ sodium selenite with or without BPE. The medium was changed on day 4 and cell extracts were taken at $\sim 90 \%$ confluence (day 7 or 8 ) by washing with $1 \mathrm{ml} \mathrm{PBS}$ and then incubating in $0.4 \mathrm{ml}$ lysis buffer $(0.5 \% \mathrm{SDS}$, $0.5 \mathrm{M}$ Tris ( $\mathrm{pH} 7.8), 50 \mathrm{mM} \mathrm{MgCl}_{2}, 1 \times$ protease inhibitor cocktail (Roche)) for $5 \mathrm{~min}$. The lysates were boiled in a water bath for 5 min, then cooled to room temperature. Subsequently, $0.048 \mathrm{ml}$ of nuclease reagent $(1 \mathrm{mg} / \mathrm{ml}$ DNase, $1 \mathrm{mg} / \mathrm{ml}$ RNase, $0.5 \mathrm{M}$ Tris ( $\mathrm{pH}$ 7.8), $50 \mathrm{mM} \mathrm{MgCl} 2,1 \times$ protease inhibitor cocktail) was added to the lysates and incubated at room temperature for $10 \mathrm{~min}$, then total protein was precipitated by the addition of $2.4 \mathrm{ml}$ cold acetone. The samples were incubated at $-20^{\circ} \mathrm{C}$ for $1 \mathrm{~h}$ or overnight, followed by centrifugation at $1000 \times g$ for $5 \mathrm{~min}$ at $4{ }^{\circ} \mathrm{C}$. Protein was resuspended in $0.06 \mathrm{ml}$ of $1 \times$ SDS-PAGE sample buffer $(50 \mathrm{mM}$ Tris- $\mathrm{HCl}$ at pH 6.8, 2\% SDS, $0.1 \%$ bromophenol blue, $10 \%$ glycerol, $0.1 \mathrm{M}$ $\beta$-mercaptoethanol).

\section{Cell viability assays}

RWPE-1, LNCaP, PC-3 prostate cells were seeded at 5000 cells/ $\mathrm{cm}^{2}$ in a total volume of $0.1 \mathrm{ml}$ into a 96-well clear bottom tissue culture assay plate containing medium with either sodium selenite, SeMet or sodium selenate at a two-fold serial dilution from either $10 \mu \mathrm{M}$ or $100 \mu \mathrm{M}$ as indicated in Figure 6. Cells were allowed to grow undisturbed for $7 \mathrm{~d}$ and then assayed for viability using the Cell Titer-Glo Luminescent Cell Viability Assay (Promega), according to the manufacturer's protocol. Luminescence was recorded in a Dynex MLX plate luminometer and the activity measured was used to represent the data as the percent viability normalized to the growth of untreated cells. The data reported are the average $( \pm$ S.E.) of three independent experiments.

\section{Western blot analysis}

Whole-cell extracts $\left(3 \%\right.$ of the protein extracted from a $9.6 \mathrm{~cm}^{2}$ well for all except those in Figures 2A, 4B and 4C where 16.6\% was used) were resolved on a $15 \%$ SDS-PAGE gel, blotted to nitrocellulose membrane (Amersham Biosciences), blocked for $1 \mathrm{~h}$ in $5 \%$ non-fat dried milk, and then cut between the 37 and $25 \mathrm{kDa}$ molecular weight markers (BioRad). The membranes with the higher weight markers were incubated either with a monoclonal anti-human eukaryotic elongation factor-1 A antibody (Upstate Biotechnology) at a 1:1 250000 dilution for PC-3 and LNCaP blots or a 1:250 000 dilution for RWPE-1 blots or with a polyclonal anti-human $\beta$-actin antibody (Santa Cruz Biotechnology Inc.) at a 1:100 000 dilution in the same blocking solution at $4{ }^{\circ} \mathrm{C}$ overnight. The membranes with 
the lower weight markers were incubated either with a monoclonal anti-human GPX-1 antibody (Stressgen Bioreagents) at a 1:1 000 dilution, a polyclonal anti-human GPX2 antibody (kindly provided by Regina Brigelius-Flohé, University of Potsdam) at 1:1 000 or with a polyclonal anti-rat GPX4 antibody (kindly provided by Donna Driscoll, Cleveland Clinic Foundation) at a 1:10 000 dilution in the same blocking solution at $4{ }^{\circ} \mathrm{C}$ overnight. Primary antibodies were detected with horseradish peroxidase-conjugated secondary antibodies (Pierce) and visualized using the SuperSignal West Femto kit (Pierce) according to the manufacturer's protocol.

\section{Northern blot analysis}

RWPE-1, LNCaP and PC-3 cells were grown in the presence or absence of $50 \mathrm{nM}$ selenite or $5 \mu \mathrm{M}$ SeMet for $7 \mathrm{~d}$. Total RNA was extracted from these cells grown in six-well plates using TRIzol (Invitrogen), according to the manufacturer's protocol. Three micrograms of RNA from each sample were resolved at $75 \mathrm{~V}$ for $4 \mathrm{~h}$ in a $1.2 \%$ agarose, $7 \%$ formaldehyde gel that was transferred to an Immobilon- $\mathrm{Ny}^{+}$membrane (Millipore) in $20 \times \mathrm{SSC}$ buffer overnight. The membrane was probed with $5.0 \times 10^{6} \mathrm{cpm}$ of $\left[\alpha^{-32} \mathrm{P}\right] \mathrm{dCTP}-\mathrm{la}-$ beled GPX1 cDNA probe made by RadPrime DNA Labeling System (Invitrogen) in ULTRAhyb (Ambion) ultrasensitive hybridization buffer at $42{ }^{\circ} \mathrm{C}$ overnight. The membrane was washed and exposed to a PhosphorImager screen overnight and visualized on a Typhoon scanner (GE Healthcare). The blot was stripped and reprobed with $\left[\alpha-{ }^{32} \mathrm{P}\right]$ dCTP-labeled GPX2, GPX4 and $\beta$-actin cDNA probes with the same conditions.

\section{Results}

GPX1 protein expression in RWPE-1 prostate cells is maximally induced by low concentrations of organic and inorganic selenium

In order to examine the precise concentration of selenium required for maximum selenoprotein expression, we cultured RWPE-1 cells in selenium-free and selenitesupplemented media. The cells were cultured for $7 \mathrm{~d}$ in selenium-free MCDB-151 media containing 12.5-200 nM selenite, then whole-cell lysates were examined for GPX1 expression by immunoblot (data not shown). Maximum GPX1 expression was observed at the lowest concentration, so we cultured cells in the presence of lower concentrations ranging from 1 to $50 \mathrm{nM}$ selenite. Figure 1 shows that GPX1 expression is substantial even in the absence of added selenium, but it does increase as a function of selenite concentration, with maximum expression occurring at $\sim 10$ $\mathrm{nM}$ contrasted with $\sim 5 \mu \mathrm{M}$ required for $50 \%$ toxicity (see below). Thus, doses as much as 500 times lower than the toxic dose are sufficient for maximum GPX1 expression in RWPE-1 prostate cells in culture. When the two other forms of selenium were tested for their ability to induce GPX1, it was found that maximum GPX1 expression occurs at $\sim 50 \mathrm{nM}$ selenate and $\sim 250 \mathrm{nM}$ SeMet (Figure 1B and 1C). These results demonstrate that RWPE-1 cells express GPX1 in the absence of added selenium, but that maximum expression requires selenium supplementation.

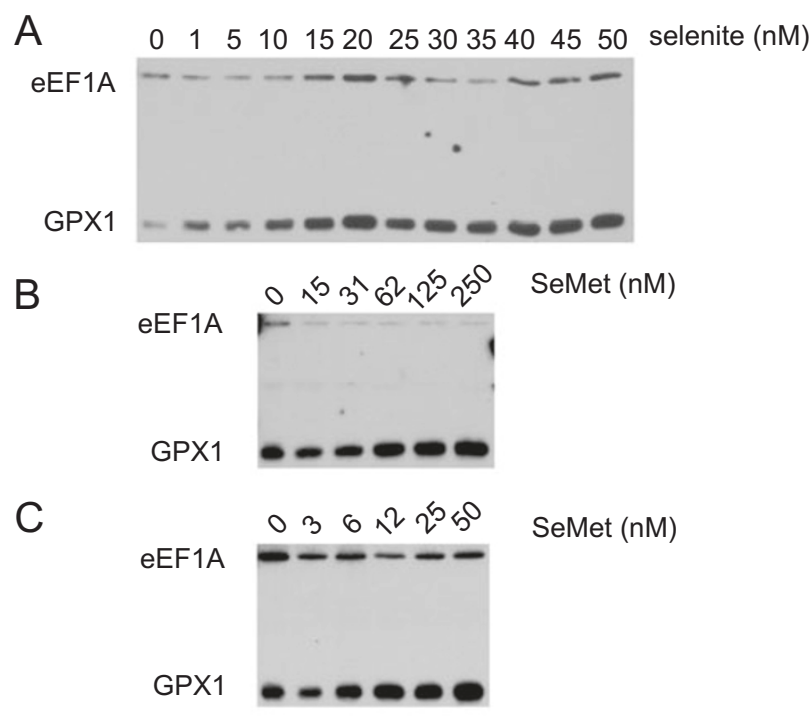

Figure 1 GPX1 expression in RWPE-1 prostate cells is induced by low concentrations of selenium. Western blot analysis of whole-cell extracts from RWPE-1 cells cultured for $7 \mathrm{~d}$ in the presence of selenite (A), SeMet (B) or selenate (C) at the concentrations indicated. Total protein was blotted to nitrocellulose and probed with anti-GPX1 and anti-eEF1A antibodies.

GPX1 protein expression in prostate cancer cells is induced by both organic and inorganic selenium

To examine whether GPX1 expression in prostate cancer cells were similarly responsive to selenium supplementation, we performed the same experiments using $\mathrm{LNCaP}$ and PC-3 cells. Figure 2 shows that GPX1 expression is dramatically induced by all forms of added selenium in both cell types, but most dramatically in PC-3 cells. In this case, maximum GPX1 expression in $\mathrm{LNCaP}$ was achieved with $\sim 30 \mathrm{nM}$ selenite, $\sim 250 \mathrm{nM}$ selenate and $\sim 125 \mathrm{nM}$ SeMet. This is in contrast to PC-3 cells where the selenite response was similar $(\sim 40 \mathrm{nM})$, but maximum induction occurred at $\sim 0.5 \mu \mathrm{M}$ for selenate and $\sim 2 \mu \mathrm{M}$ for SeMet. These differences provide evidence that prostate cancer cells are fundamentally altered in their ability to utilize exogenous selenium in a compound-dependent fashion.

The fact that LNCaP and especially PC-3 cells are limited in their ability to express GPX1 protein in the absence of added selenium led us to believe that the serum used for this study was deficient in a form of selenium usable by these cells. Interestingly, RWPE-1 cells showed much lower levels of GPX1 induction, leading us to believe that the culture conditions for these cells (MCDB-151 with BPE and purified EGF) may be providing a source of selenium that was not available in the medium that was used to culture LNCaP and PC-3 cells (RPMI with $10 \%$ fetal bovine serum). Since both of the synthetic salt solutions were 
A

015101520253035404550 selenite (nM)

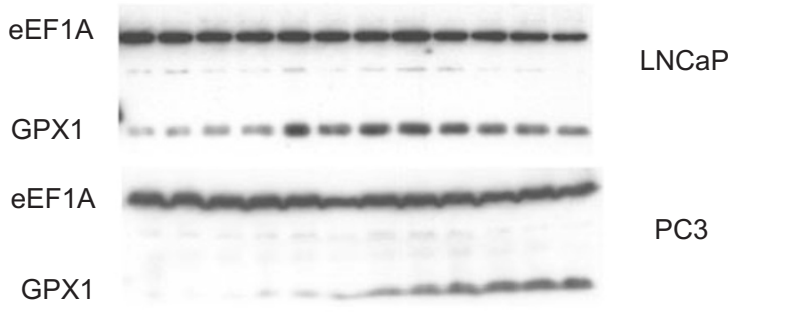

B

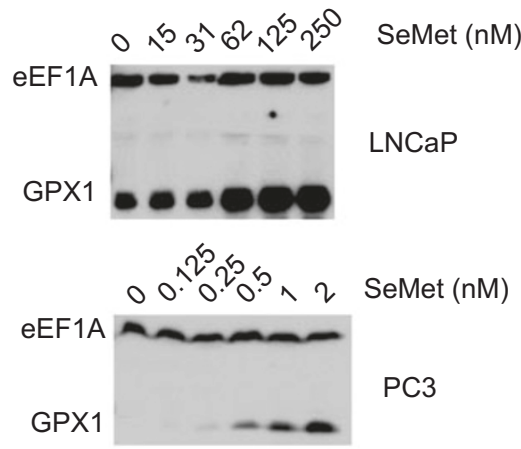

C

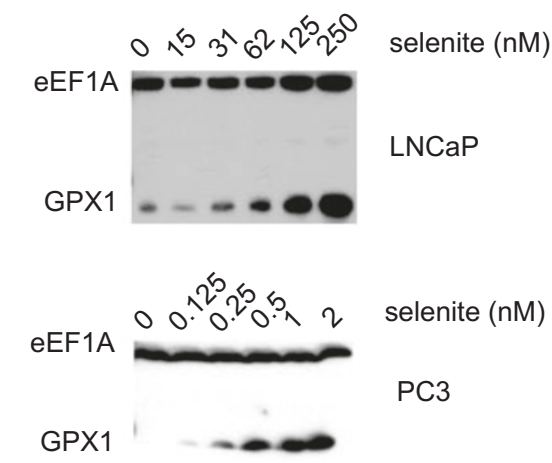

Figure 2 GPX1 expression is dramatically induced by selenium in prostate cancer cells. Western blot analysis of whole-cell extracts from $\mathrm{LNCaP}$ and $\mathrm{PC}-3$ cells cultured for $7 \mathrm{~d}$ in the presence of selenite (A), SeMet (B) or selenate (C) at the concentrations indicated. Total protein was blotted to nitrocellulose and probed with anti-GPX1 and anti-eEF1A antibodies.

selenium-free, we expected that the BPE may have been supplying usable selenium to the RWPE-1 cells. To test this hypothesis, PC-3 cells were also grown in the presence of the same concentration of BPE as that used to culture RWPE-1 cells. Surprisingly, as shown in Figure 3, BPE was not able to induce GPX1 expression at all, suggesting that in the case of RWPE-1 cells, they are able to utilize the selenium compounds found in the BPE while cancer cells cannot. The same result was obtained when EGF was included as well (data not shown). These results may indicate a significant difference between cells derived from prostate cancer and those derived from normal epithelium in their ability to utilize sources of organic selenium.

GPX1 levels are reduced in PC-3 but not LNCaP cells

The above data suggest that the overall levels of GPX1 are quite different even when selenium levels are sufficient. To directly address this question, all three prostate cell lines were cultured for at least $7 \mathrm{~d}$ either in the absence of selenium (Figure 4A, lanes 1-3) or in the presence of excess but subtoxic levels of selenite (50 nM; lanes 4-6), selenate (1 $\mu \mathrm{M}$; lanes 7-9) and SeMet (5 $\mu \mathrm{M}$; lanes 10-12). While Western blots are not highly quantitative, densitometric analysis of the blot shows that relative to RWPE-1 cells, GPX1 levels are elevated in LNCaP cells and drastically reduced in PC-3 cells (Figure 4A, bottom panel). The expression of GPX1 was analyzed as a function of $\beta$-actin in this case because eEF1A is dramatically upregulated in LNCaP and PC-3 cells (data not shown), consistent with previous studies demonstrating the upregulation of eEF1A in cancer cells [18]. These data suggest a fundamental difference in selenoprotein expression between $\mathrm{LNCaP}$ and PC-3 cells.

\section{GPX4 induction is similar to that of GPXI}

Since it has been recently shown that the two serum selenoproteins (Sel P and GPX 3) are maximally expressed at different levels of supplemental selenium [19], we wanted to determine if two selenoproteins were induced to the same extent at the same level of selenium supplementation in prostate cells. To that end, the samples used to examine GPX1 levels were also examined for GPX4 expression via

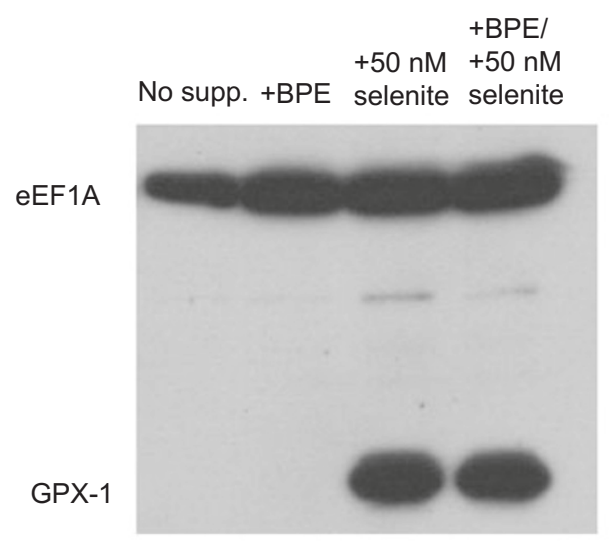

Figure 3 BPE does not induce GPX1 expression in prostate cancer cells. PC-3 cells were grown in the presence or absence of BPE and $50 \mathrm{nM}$ sodium selenite and whole-cell extracts were analyzed by immunoblot. Total protein was blotted to nitrocellulose and probed with anti-GPX1 and anti-eEF1A antibodies. 
Western blot analysis. Figure 4B shows that maximal GPX4 expression occurs at the same ranges for all three selenium compounds in PC-3 cells when compared to GPX1 induction (compare Figures 2-4B). These data show that at least for two members of the GPX family, selenium induction of expression is the same for both genes, suggesting that there is no difference in Sec-tRNA ${ }^{[\mathrm{Ser}] \mathrm{Sec}}$ utilization between these two selenoproteins in this cell type.

\section{GPX2 expression is only detected in RWPE-1 prostate cells}

To examine the epithelial cell-specific selenoprotein GPX2 induction, all three prostate cell lines were grown in the presence or absence of selenite ( 5 or $20 \mathrm{nM}$ ). Figure $4 \mathrm{C}$ shows that GPX2 levels were not detectable in PC-3 or $\mathrm{LNCaP}$ cells, even in the presence of added selenium. In addition, and consistent with GPX1, selenium supple- mentation increased GPX2 levels only by about a factor of $\sim 2$. The expression of GPX2 is distinct from that of GPX1 and GPX4 and provides evidence that individual selenoproteins are regulated independently during prostate carcinogenesis.

\section{Selenoprotein $m R N A$ expression is also regulated in pros- tate cells}

To address the extent to which mRNA levels contribute to the differences observed in GPX protein levels, Northern analysis was performed on RNA samples derived from cells cultured in the presence and absence of supplemental selenite and SeMet (RNA samples derived from selenatetreated cells were inexplicably but reproducibly insoluble after extraction). Figure 5 shows that for GPX1, the mRNA levels were consistently lower in non-supplemented cells but higher in supplemented cells, especially in PC-3 cells.
A
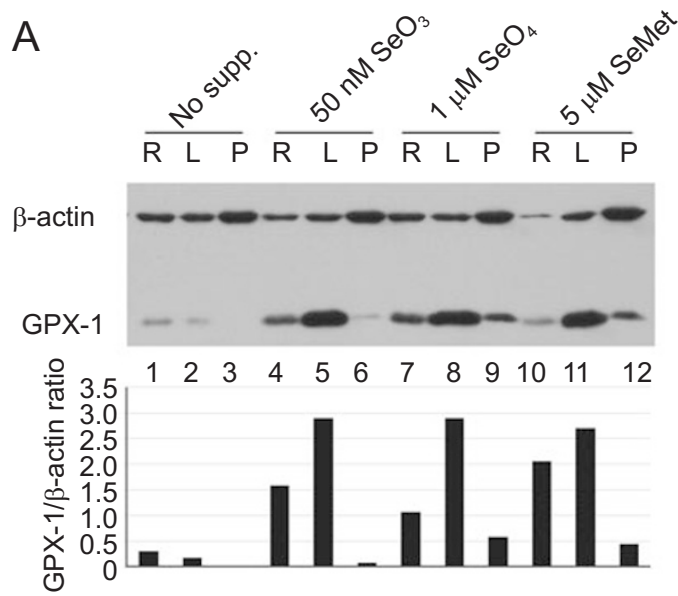

C

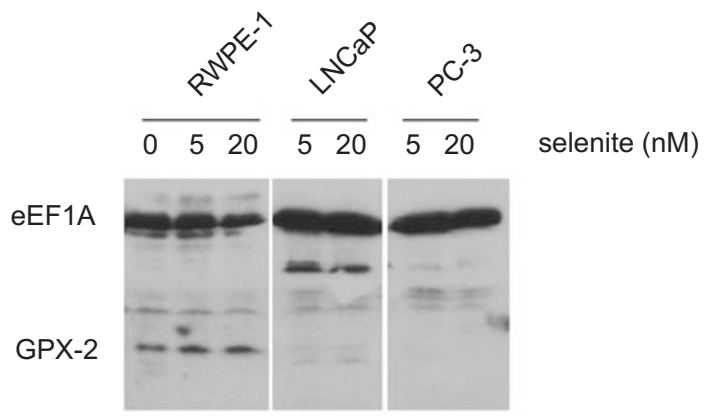

B selenite $(\mathrm{nM})$
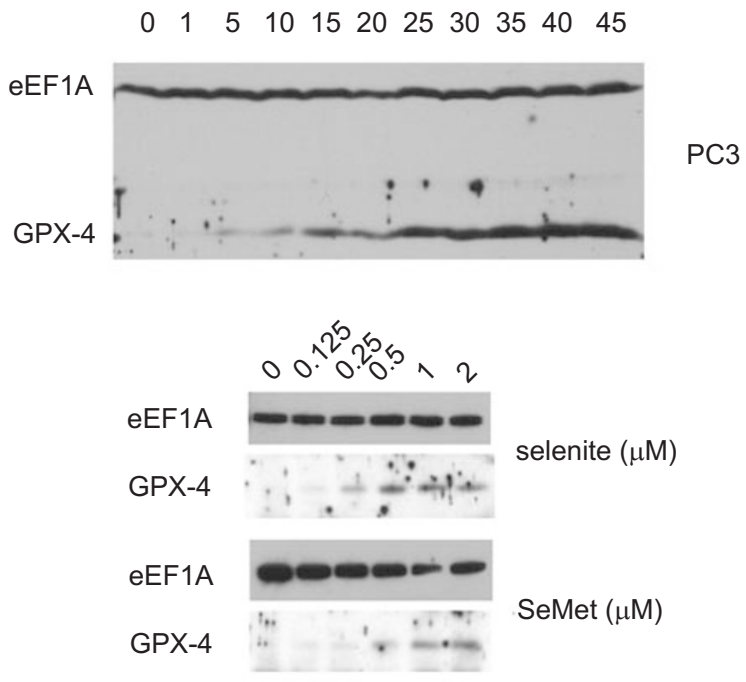

Figure 4 (A) GPX1 levels are different in RWPE-1 and prostate cancer cells even when selenium levels are sufficient. Western blot analysis of whole-cell extracts from RWPE-1, LNCaP and PC-3 cells cultured for $7 \mathrm{~d}$ in the presence of selenite, SeMet or selenate at the concentrations indicated. Total protein was blotted to nitrocellulose and probed with anti-GPX1 and anti-human b-actin antibodies. The ratio of GPX1 to b-actin expression was determined by densitometry and shown in graphical form in the bottom panel. (B) GPX4 protein expression is similar to that of GPX1 expression. Western blot analysis of total protein extracted from PC-3 cells cultured for $7 \mathrm{~d}$ in the presence of selenite (top panel), selenate (middle panels) or SeMet (bottom panels) at the concentrations indicated. Total protein was blotted to nitrocellulose and probed with anti-GPX4 and anti-eEF1A antibodies. (C) Western blot analysis of whole-cell extracts from RWPE-1, LNCaP and PC-3 cells cultured for $7 \mathrm{~d}$ in the presence or absence of 5 or $20 \mathrm{nM}$ sodium selenite. Total protein was blotted to nitrocellulose and probed with anti-GPX2 and anti-eEF1A antibodies. 


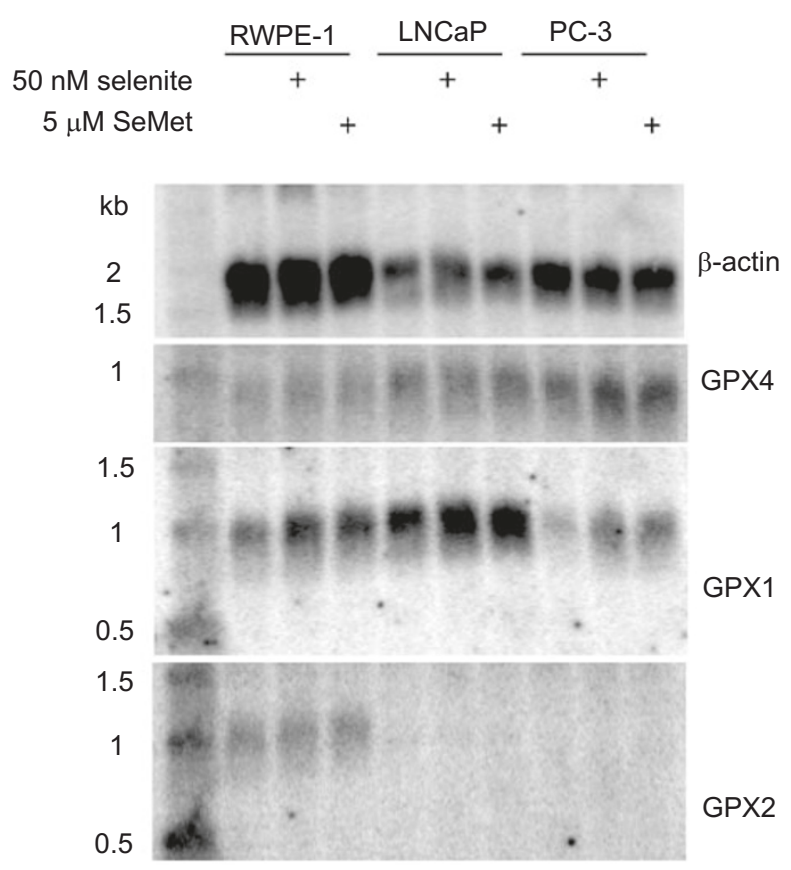

Figure 5 Northern blot analysis of selenoprotein mRNA expression as a function of selenium supplementation. RWPE-1, LNCaP, and PC-3 cells were grown in the presence or absence of $50 \mathrm{nM}$ selenite or 5 $\mu \mathrm{M}$ SeMet for $7 \mathrm{~d}$. Total mRNA was examined by Northern analysis with probes designed to detect b-actin, GPX1, GPX2 and GPX4.

This is consistent with the protein levels observed in Figure $4 \mathrm{~A}$ and the previous reports showing that GPX $1 \mathrm{mRNA}$ is unstable when selenium is deficient [21-24]. GPX4 mRNA levels are not as consistent with protein levels since clear expression is detectable even in PC-3 cells under low selenium conditions where GPX4 protein is lacking, indicating a clear case of regulation at the translational level. GPX2 mRNA levels were directly related to protein levels as they were undetectable in the prostate cancer cells, suggesting that the downregulation of this gene in prostate cancer occurs at the level of transcription or a dramatic decrease in RNA stability.

LNCaP and PC-3 prostate cancer cells are not more sensitive to selenium toxicity than RWPE-1 cells

In order to compare the levels of selenium required for GPX induction with those required for toxicity, we wished to establish the levels of selenium that induced a chronic toxic effect as measured by cell viability. To that end, RWPE-1, LNCaP and PC-3 cells were grown in varying concentrations of sodium selenite (selenite), sodium selenate (selenate) or SeMet for at least $7 \mathrm{~d}$ before determining viability in a luciferase-based assay (see Materials and Methods). To determine the point at which each compound displayed toxicity, we used a range of 6.25 to $100 \mu \mathrm{M}$ - far exceeding the $\sim 2.5 \mu \mathrm{M}$ blood level found in patients taking supplemental selenium [2]. Surprisingly, under these conditions, RWPE-1 cells are the most sensitive to all three compounds, indicating that under these conditions and with these cell lines, we are unable to reproduce the findings that prostate cancer cells are more sensitive to selenite than non-tumorigenic lines. Consistent with previous studies, however, only sodium selenite showed toxicity at near-physiological levels [20]. To further investigate toxicity in the physiological range, cells were treated with lower doses of all three compounds ranging from 0.62 to $10 \mu \mathrm{M}$ (Figure 6B). In this case only selenite-induced toxicity, but this is only evident in RWPE-1 prostate cells. Interestingly, selenite seems to specifically enhance RWPE-1 growth at lower doses, but this effect is unlikely to stem from selenoprotein induction since all three compounds are able to stimulate selenoprotein expression in the concentration range below that required for toxicity (see above). Together, these results describe the range of selenium concentrations that do not reduce cell viability, thus confirming that in prostate cell lines the concentration of selenium required for maximum GPX expression is well below that required for toxicity.

\section{Discussion}

One of the central questions regarding selenium and prostate cancer is whether selenoproteins are involved or not. The fact that selenoproteins are dramatically regulated differentially when comparing non-tumorigenic versus cancer cells suggests that this is the case, but whether this is cause of or result from cell transformation remains to be determined. In addition, the regulation of selenoproteins and the chemopreventive effects of selenium may be unrelated phenomena. In this work, we have begun to address these questions by examining the regulation of selenoprotein expression as a function of selenium supplementation in non-tumorigenic and cancerous cells. We have demonstrated that not only is selenoprotein expression affected, but selenium utilization also becomes compromised in PC-3 and not LNCaP cells. Thus, changes in selenoprotein expression and selenium utilization may provide valuable markers for prostate cancer progression. Further work in the development of a chemoprevention model system will be required to discern the precise role of selenoprotein in preventing either prostate cancer initiation, progression or both.

Our results indicate that prostate cancer cells are viable in the presence of supplemental selenium in the physiological range, thus providing further evidence that the chemo- 

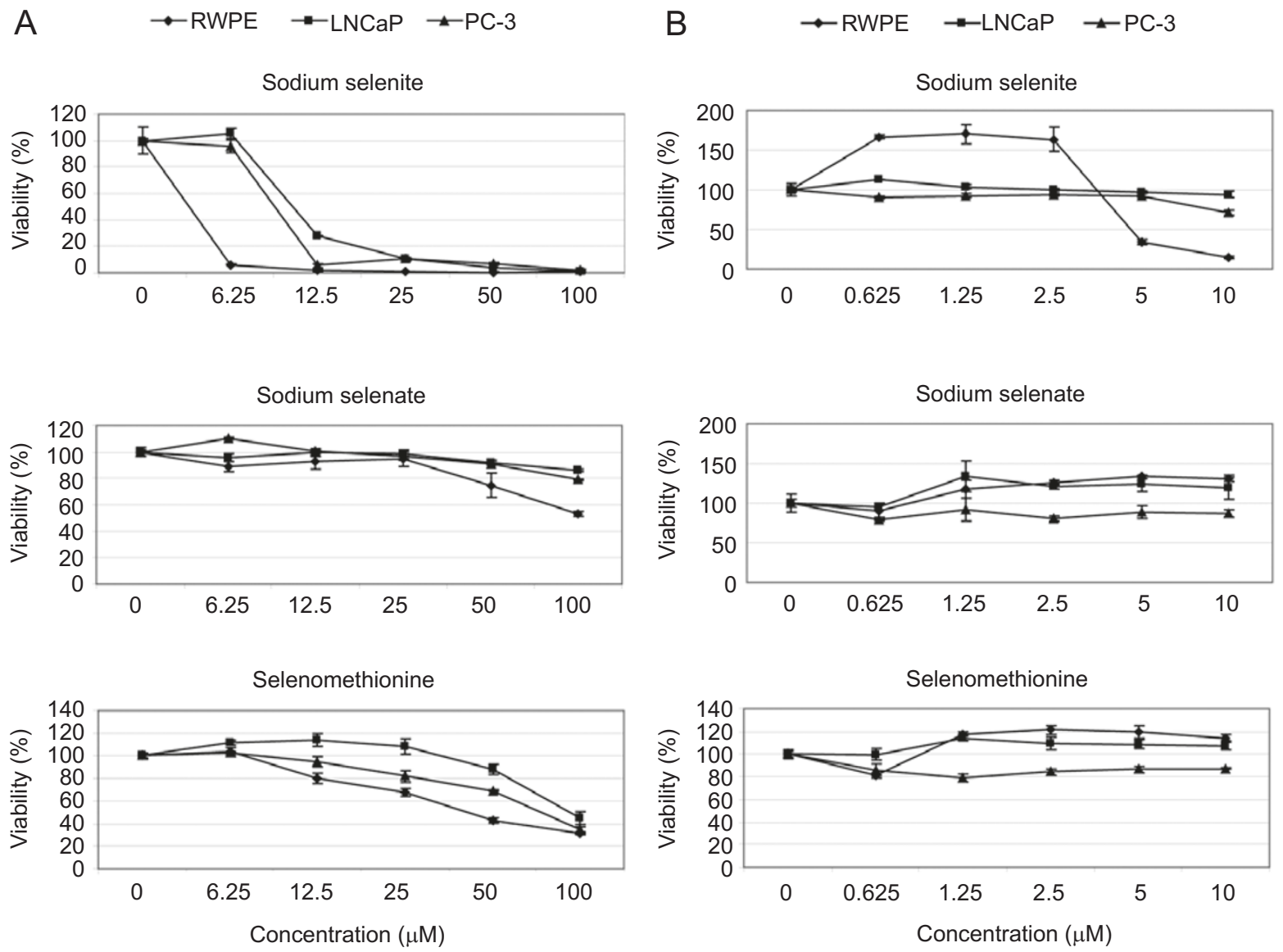

Figure 6 Cell viability as a function of selenium supplementation. RWPE-1 and cancerous (LNCaP, PC-3) prostate cells were cultured for $7 \mathrm{~d}$ in the presence of high (A) or low (B) levels of the selenium compounds at the indicated concentrations. Cell viability was assessed using a luminescent ATP release assay. The data are represented as the percent viability normalized to the growth of untreated cells. Each data point represents the mean of three experiments $( \pm$ S.E.).

protective effect of selenium supplementation observed in the population may be based in large part on selenoprotein expression and not on the selective pro-apoptotic activity of excess dietary selenium. One possible explanation for this result is that prostate cells in culture are not competent to properly metabolize the selenium compounds used in this study. While this is certainly possible, we have demonstrated here that the cell lines used are able to efficiently metabolize inorganic and organic selenium for Sec synthesis. This suggests that the sulfur/selenium metabolism pathways (production of selenide from both organic and inorganic sources) are active in these cells. Interestingly, we find distinct differences in selenium utilization. RWPE-1 cells express well over $50 \%$ of their maximum levels of GPX1 without selenium supplementation while PC-3 cells show little or no GPX1 expression in the absence of added selenium. This suggests that RWPE- 1 cells are able to utilize the selenium found in the culture medium. Since all cell types were cultured in selenium-free salt solutions, the only source of selenium would be found in the undefined supplements (BPE for RWPE-1 and 10\% calf serum for PC-3). The fact that PC-3 and LNCaP cells showed no induction of GPX1 expression even when supplemented with BPE suggests that naturally occurring sources of animal-derived selenium (most likely protein-bound SeMet) are unavailable to prostate cancer cells. The reduced ability of cancer cells to utilize selenium may shed significant light on the mechanism behind the reduction in selenoprotein expression during carcinogenesis. These data also underscore the fact that selenoproteins may not be optimally expressed in many standard tissue culture conditions, a phenomenon that should be carefully considered when studying the effects of oxidative stress on cells in culture.

In this study, we have observed several modes of selenoprotein regulation. First and least surprising is the fact that selenoprotein translation is significantly stimulated 
by selenium supplementation. This is best illustrated by GPX4 expression in PC-3 cells where mRNA is present but not translated in the absence of added selenium. This is also true for GPX1, but to a lesser extent, because mRNA levels in the absence of selenium supplementation are reduced. This is very likely to be the result of lower mRNA stability as has been observed for GPX1 in multiple studies [21-24]. In the case of GPX2, the expression observed appears to be dramatically regulated at the RNA level, most likely at the level of transcription because its mRNA is not detectable even in the presence of supplemental selenium. This is in agreement with a recent study of prostate cancer progression in the Nkx3.1 mouse model where loss of Nkx3.1 expression resulted in reduced GPX2 RNA levels and unchanged GPX1 RNA levels [25]. These results together with those described in this work describe the complexity of selenoprotein regulation in the prostate and provide the groundwork for future studies designed to dissect the roles of individual selenoproteins in proper prostate cell function.

Perhaps the most surprising result from these studies is the higher sensitivity of RWPE-1 cells versus transformed cells to the physiological range of selenium in the form of selenite. The significance of this is, of course, somewhat limited by the artificial nature of cell culture and the limited number of cell lines used in this study. In previous studies, the toxicity of selenite for LNCaP cells was greater. For example, maximum cytotoxicity was observed at $2.5 \mu \mathrm{M}$ in one case [26], and maximum caspase expression was achieved at $4 \mu \mathrm{M}$ in another case [5]. The most likely explanations for these discrepancies are the length of treatment (our cells were treated for $7 \mathrm{~d}$ and may have experienced some adaptation to the selenite) and/or the type of viability assay used. Since neither of these previous reports included a direct comparison with "normal" cells, it is difficult to assess the full extent of the discrepancy. Interestingly, the most recent report regarding the selective sensitivity of primary prostate cancer cells indicated that $25 \mu \mathrm{M}$ selenite was the minimum dose of selenite required for toxicity [27]. Our data, therefore, further call into question the universality of the idea that prostate cancer cells are inherently more sensitive to the pro-apoptotic effects of selenium, thus indirectly providing support for the idea that selenoprotein activity is sufficient to explain the chemoprotective effects reported in clinical studies.

\section{Acknowledgments}

We thank Terri Kinzy, Stephane Gross and Cory AbateShen for critiques. We also thank Regina Brigelius-Flohe for providing anti-GPX2 antibody. This work was funded by the Dean and Betty Gallo Prostate Cancer Center at the Cancer Institute of New Jersey with funds obtained from the Department of Defense (PRC) and by a CR Bard Foundation fellowship (FJPIII).

\section{References}

1 Clark LC, Combs GF Jr, Turnbull BW, et al. Effects of selenium supplementation for cancer prevention in patients with carcinoma of the skin. A randomized controlled trial. Nutritional Prevention of Cancer Study Group. JAMA 1996; 276:1957-1963.

2 Duffield-Lillico AJ, Reid ME, Turnbull BW, et al. Baseline characteristics and the effect of selenium supplementation on cancer incidence in a randomized clinical trial: a summary report of the Nutritional Prevention of Cancer Trial. Cancer Epidemiol Biomarkers Prev 2002; 11:630-639.

3 Duffield-Lillico AJ, Slate EH, Reid ME, et al. Selenium supplementation and secondary prevention of nonmelanoma skin cancer in a randomized trial. J Natl Cancer Inst 2003; 95:1477-1481.

4 Jiang C, Kim KH, Wang Z, Lu J. Methyl selenium-induced vascular endothelial apoptosis is executed by caspases and principally mediated by p38 MAPK pathway. Nutr Cancer 2004; 49:174-183.

5 Jiang C, Hu H, Malewicz B, Wang Z, Lu J. Selenite-induced p53 Ser-15 phosphorylation and caspase-mediated apoptosis in LNCaP human prostate cancer cells. Mol Cancer Ther 2004; 3:877-884.

6 Ip C, Thompson HJ, Zhu Z, Ganther HE. In vitro and in vivo studies of methylseleninic acid: evidence that a monomethylated selenium metabolite is critical for cancer chemoprevention [in process citation]. Cancer Res 2000; 60:2882-2886.

7 Diwadkar-Navsariwala V, Diamond AM. The link between selenium and chemoprevention: a case for selenoproteins. J Nutr 2004; 134:2899-2902.

8 Lu J, Jiang C. Selenium and cancer chemoprevention: hypotheses integrating the actions of selenoproteins and selenium metabolites in epithelial and non-epithelial target cells. Antioxid Redox Signal 2005; 7:1715-1727.

$9 \mathrm{Hu}$ YJ, Korotkov KV, Mehta R, et al. Distribution and functional consequences of nucleotide polymorphisms in the 3'-untranslated region of the human Sep15 gene. Cancer Res 2001; 61:23072310.

10 Kumaraswamy E, Malykh A, Korotkov KV, et al. Structureexpression relationships of the $15-\mathrm{kDa}$ selenoprotein gene. Possible role of the protein in cancer etiology. J Biol Chem 2000; 275:35540-35547.

11 Zachara BA, Szewczyk-Golec K, Tyloch J, et al. Blood and tissue selenium concentrations and glutathione peroxidase activities in patients with prostate cancer and benign prostate hyperplasia. Neoplasma 2005; 52:248-254.

12 Gladyshev VN, Factor VM, Housseau F, Hatfield DL. Contrasting patterns of regulation of the antioxidant selenoproteins, thioredoxin reductase, and glutathione peroxidase, in cancer cells. Biochem Biophys Res Commun 1998; 251:488-493.

13 Irons R, Carlson BA, Hatfield DL, Davis CD. Both selenoproteins and low molecular weight selenocompounds reduce colon cancer risk in mice with genetically impaired selenoprotein expression. J Nutr 2006; 136:1311-1317.

14 Caban K, Copeland PR. Size matters: a view of selenocysteine incorporation from the ribosome. Cell Mol Life Sci 2006; 63:7381. 
15 Driscoll DM, Copeland PR. Mechanism and regulation of selenoprotein synthesis. Annu Rev Nutr 2003; 23:17-40.

16 Hatfield DL, Gladyshev VN. How selenium has altered our understanding of the genetic code. Mol Cell Biol 2002; 22:35653576.

17 Bello D, Webber MM, Kleinman HK, Wartinger DD, Rhim JS. Androgen responsive adult human prostatic epithelial cell lines immortalized by human papillomavirus 18. Carcinogenesis 1997; 18:1215-1223

18 Chen L, Madura K. Increased proteasome activity, ubiquitinconjugating enzymes, and eEF1A translation factor detected in breast cancer tissue. Cancer Res 2005; 65:5599-5606.

19 Xia Y, Hill KE, Byrne DW, Xu J, Burk RF. Effectiveness of selenium supplements in a low-selenium area of China. Am J Clin Nutr 2005; 81:829-834.

20 Menter DG, Sabichi AL, Lippman SM. Selenium effects on prostate cell growth. Cancer Epidemiol Biomarkers Prev 2000; 9:1171-1182.

21 Moriarty PM, Reddy CC, Maquat LE. Selenium deficiency reduces the abundance of mRNA for Se-dependent glutathione peroxidase 1 by a UGA-dependent mechanism likely to be nonsense codon-mediated decay of cytoplasmic mRNA. Mol Cell
Biol 1998; 18:2932-2939

22 Sun X, Moriarty PM, Maquat LE. Nonsense-mediated decay of glutathione peroxidase $1 \mathrm{mRNA}$ in the cytoplasm depends on intron position. EMBO J 2000; 19:4734-4744.

23 Sun X, Li X, Moriarty PM, Henics T, LaDuca JP, Maquat LE. Nonsense-mediated decay of mRNA for the selenoprotein phospholipid hydroperoxide glutathione peroxidase is detectable in cultured cells but masked or inhibited in rat tissues. Mol Biol Cell 2001; 12:1009-1017.

24 Weiss Sachdev S, Sunde RA. Selenium regulation of transcript abundance and translational efficiency of glutathione peroxidase1 and -4 in rat liver. Biochem $\mathrm{J} 2001 ; \mathbf{3 5 7}: 851-858$.

25 Ouyang X, DeWeese TL, Nelson WG, Abate-Shen C. Loss-offunction of $\mathrm{Nkx} 3.1$ promotes increased oxidative damage in prostate carcinogenesis. Cancer Res 2005; 65:6773-6779.

26 Zhong W, Oberley TD. Redox-mediated effects of selenium on apoptosis and cell cycle in the LNCaP human prostate cancer cell line. Cancer Res 2001; 61:7071-7078.

27 Husbeck B, Nonn L, Peehl DM, Knox SJ. Tumor-selective killing by selenite in patient-matched pairs of normal and malignant prostate cells. Prostate 2006; 66:218-225. 